\title{
Removal of Sodium Dodecyl Sulfate Surfactant from Aqueous Dispersions of Single-Wall Carbon Nanotubes
}

Jamie E. Rossi, ${ }^{1,2}$ Karen J. Soule, ${ }^{1,2}$ Erin Cleveland, ${ }^{3}$ Scott W. Schmucker, ${ }^{3}$ Cory D. Cress, ${ }^{3}$ Nathanael D. Cox, ${ }^{1,2,4}$ Andrew Merrill, ${ }^{1,2}$ Brian J. Landi ${ }^{1,2 *}$

${ }^{1}$ Department of Chemical Engineering, Rochester Institute of Technology, Rochester, New York 14623, USA

${ }^{2}$ NanoPower Research Laboratory, Rochester Institute of Technology, Rochester, NY 14623, USA

${ }^{3}$ Electronics Science and Technology Division, United States Naval Research Laboratory, Washington D.C. 20375, USA

${ }^{4}$ Department of Microsystems Engineering, Rochester Institute of Technology, Rochester, New York 14623, USA

\section{Abstract:}

A reagent-based treatment method was developed for the removal of sodium dodecyl sulfate (SDS) from aqueous dispersions of single-wall carbon nanotubes (SWCNTs). Based on a survey of various reagents, organic solvents emerged as the most effective at interrupting the SDS:SWCNT interaction without producing deleterious side reactions or causing precipitation of the surfactant. Specifically, treatment with acetone or acetonitrile allows for the facile isolation of SWCNTs with near complete removal of SDS through vacuum filtration, resulting in a 100x reduction in processing time. These findings were validated via quantitative analysis using thermogravimetric analysis, Raman spectroscopy, 4-point probe electrical measurement, and xray photoelectron spectroscopy. Subsequent thermal oxidation further enhances the purity of the reagent treated samples and yields bulk SWCNT samples with $>95 \%$ carbonaceous purity. The proposed reagent treatment method thus demonstrates potential for large volume SWCNT processing.

Keywords: Dispersion, solvent interaction, SWCNT purity, surfactant removal

*Corresponding Author: Tel: 585 475-4726, Email: brian.landi@ rit.edu (Brian J. Landi) 


\section{Introduction:}

Amphiphilic surfactants have facilitated the use of single-wall carbon nanotubes (SWCNTs) in a number of applications, enabling the exploration of their unique physical and electrical properties. The limited solubility of SWCNTs in water makes them difficult to work with, necessitating the use of surfactants and ultrasonication to form stable aqueous dispersions. Sodium dodecyl sulfate (SDS) is the most commonly employed surfactant in applications such as spectroscopy [1-5], chirality separations [6-8], and device fabrication (i.e., transparent conducting films [9, 10], organic photovoltaics [11], batteries [12], etc.). However, the presence of residual SDS on the surface of the SWCNTs makes it difficult to probe their intrinsic properties and can require additional post-processing purification for some applications.

Early reports indicated that SDS could be removed from SWCNTs via vacuum filtration and copious water rinsing $[13,14]$. The amount of water rinsing was typically determined using visual indicators when bubbling of the surfactant through the filter membrane stopped; however, there has been insufficient evidence of the resulting SWCNT purity through quantitative analysis for this method. In fact, recent literature demonstrates that simple water rinsing alone leaves behind up to 15 wt. \% residual SDS in SWCNT thin-films prepared by this technique [12]. As such, the addition of thermal oxidation and/or annealing after sample processing facilitates the removal of residual SDS from water rinsed SWCNT thin-films and bulk papers [15-18], yet complete SDS removal with thermal treatments alone has not been demonstrated. Alternate approaches have examined the removal of residual SDS and other common surfactants by soaking SWCNT samples in concentrated nitric acid, which is proposed to remove the residual SDS, as well as defective SWCNTs from the sample [9-12, 19]. Furthermore, acidification of the surfactant salt with $\mathrm{HCl}$ creates a byproduct that can be solubilized and washed away with 
ethanol [20]. More sophisticated techniques have focused on the decomposition of organic compounds, such as surfactants, through the use of the Fenton reaction [21]; however, as with the acid treatments this is a time consuming process that has not been shown to fully remove the residual SDS from the SWCNTs.

In the current work, a facile and scalable reagent-based method of surfactant removal is developed to isolate SWCNTs from aqueous SDS-based dispersions. Various reagents are evaluated for their ability to interrupt the SDS:SWCNT interaction in solution, with the goal of precipitating the SWCNTs for easy isolation via vacuum filtration. The resulting bulk SWCNT papers were characterized using scanning electron microscopy (SEM), Raman spectroscopy, thermogravimetric analysis (TGA), 4-point probe electrical measurements, and x-ray photoelectron spectroscopy (XPS) to evaluate the purity of the reagent treated samples compared to a control that was filtered from SDS and rinsed only with water. A portion of each sample is subsequently exposed to thermal oxidation treatment in air, and the characterization is repeated to evaluate any enhanced purity of the reagent treated SWCNT samples. A distinct relationship is observed between SWCNT purity and reagent composition, and an optimized purification protocol is developed for the removal of SDS from SWCNTs.

\section{Experimental:}

\subsection{SWCNT Synthesis and Preparation of P-SWCNT Control Sample}

Single-wall carbon nanotubes (SWCNTs) were synthesized in-house via pulsed laser vaporization employing an Alexandrite laser $(755 \mathrm{~nm})$ in argon carrier gas at $1150^{\circ} \mathrm{C}$. The laser pulse was rastered over the surface of a graphite (Alfa Aesar, Graphite Flake, median 7-10 $\mu \mathrm{m}$, 99\% metal basis) target doped with 3\% w/w Ni (Sigma-Aldrich, $<1 \mu \mathrm{m}, 99.8 \%$ trace metals 
basis) and 3\% w/w Co (Sigma-Aldrich, $2 \mu \mathrm{m}, 99.8 \%$ trace metals basis) [22]. The as-produced SWCNT soot was purified of metal catalyst and carbonaceous impurities using previously established acid reflux and thermal oxidation (ramp-stop at $10^{\circ} \mathrm{C} / \mathrm{min}$ to $520^{\circ} \mathrm{C}$ ) procedures $[23$, 24]. The SWCNT sample was further purified by soaking in $37 \% \mathrm{HCl}$ for $10 \mathrm{~min}$, rinsed with copious amounts of deionized (DI) $\mathrm{H}_{2} \mathrm{O}$, then soaked for an additional $1 \mathrm{~h}$ in $37 \% \mathrm{HCl}$ to remove residual $\mathrm{Ni}$ and Co catalyst. The sample was rinsed with $\mathrm{DI}_{2} \mathrm{O}$, then thermally oxidized as described previously to remove residual acid and carbonaceous impurities. The purified SWCNT material (P-SWCNT) serves as the experimental control for all subsequent reagenttreated SWCNT samples.

\subsection{SWCNT Characterization}

The purity of the SWCNT bulk papers was analyzed qualitatively via scanning electron microscopy (Hitachi S900 Field Emission SEM, 2 kV accelerating voltage) and quantitatively using optical absorption spectroscopy (Perkin-Elmer Lambda 900), Raman spectroscopy (Jobin Yvon Horiba LabRam Spectrometer, $1.96 \mathrm{eV}$ laser energy), thermogravimetric analysis (TA Instruments TGA Q5000, Balance Purge: $\mathrm{N}_{2}(\mathrm{~g}) 20 \mathrm{~mL} / \mathrm{min}$, Sample Purge: Air $20 \mathrm{~mL} / \mathrm{min}$, Ramp Rate: $10^{\circ} \mathrm{C} / \mathrm{min}$ ), and x-ray photoelectron spectroscopy (ThermoScientific Monochromatic Al k-alpha source $(1487 \mathrm{eV}), 50 \mathrm{eV}$ pass energy, $400 \mu \mathrm{m}$ spot). Electrical characterization was performed via the 4-point probe van der Pauw method using a Signatone H100 Series probe station affixed with a NI PXI-4130 Power SMU to supply the current and PXI-4171 for voltage measurement.

\subsection{Preparation of SDS-SWCNT and $\mathrm{H}_{2} \mathrm{O}-\mathrm{SWCNT}$ Control Samples}


P-SWCNTs were dispersed in $840 \mathrm{~mL}$ of an aqueous solution of $2.0 \mathrm{wt} \%$ sodium dodecyl sulfate (SDS, Sigma-Aldrich, ACS reagent, $\geq 99.0 \%$ ) with a SWCNT concentration of $50 \mu \mathrm{g} / \mathrm{mL}$ via bath $(3 \mathrm{~h})$ and horn $(5 \mathrm{~h})$ ultrasonication in an ice-water bath. A $120 \mathrm{~mL}$ aliquot was removed, and the SWCNTs were isolated via vacuum filtration onto a PTFE membrane $(0.1 \mu \mathrm{m}$ pore size). As a control, the SWCNTs were rinsed with $50 \mathrm{~mL}$ of DI $\mathrm{H}_{2} \mathrm{O}$ and remained under vacuum in the filtration apparatus to dry for $1 \mathrm{~h}$, yielding a bulk paper (SDS-SWCNT) with an areal mass density of $0.6 \mathrm{mg} / \mathrm{cm}^{2}$. For consistency, the SWCNT mass and areal density was fixed for all additional SWCNT samples fabricated in the current study. To control for the effect of processing on the SWCNT physical and electrical properties, a second control sample was prepared whereby P-SWCNTs were ultrasonicated in DI $\mathrm{H}_{2} \mathrm{O}$ (no surfactant) using the same concentration, processing times, and conditions as described above. This sample (referred to as $\mathrm{H}_{2} \mathrm{O}-\mathrm{SWCNT}$ ) was subsequently filtered, rinsed, and dried in a manner consistent with the SDSSWCNT sample. After releasing the SDS-SWCNT and $\mathrm{H}_{2} \mathrm{O}-\mathrm{SWCNT}$ samples from the filter membrane, both samples were further dried in a vacuum oven at $100^{\circ} \mathrm{C}$ for $1 \mathrm{~h}$.

\subsection{Preparation of HCl-SWCNT Control Sample}

A third control sample (referred to as $\mathrm{HCl}-\mathrm{SWCNT}$ ) was prepared using a previously reported procedure for removing sodium deoxycholate from SWCNTs [20]. In short, a $120 \mathrm{~mL}$ aliquot of SWCNTs in $2.0 \mathrm{wt} \%$ SDS was removed from the stock and mixed with an equal volume of $6 \mathrm{M} \mathrm{HCl}$. The mixture was bath sonicated for $1 \mathrm{~h}$, then vacuum filtered onto a PTFE membrane and rinsed with $50 \mathrm{~mL}$ of $\mathrm{DI} \mathrm{H}_{2} \mathrm{O}$. The sample was redispersed in ethanol via bath sonication for $1 \mathrm{~h}$. The resulting dispersion was heated to $80^{\circ} \mathrm{C}$ in a water bath for $1 \mathrm{~h}$, and subsequently filtered onto a PTFE membrane to isolate the treated SWCNTs. The HCl-SWCNT 
sample was rinsed with fresh ethanol and remained under vacuum in the filtration apparatus to dry for $1 \mathrm{~h}$, then dried at $100^{\circ} \mathrm{C}$ for $1 \mathrm{~h}$ in a vacuum oven after releasing from the filter membrane.

\subsection{Reagent Treatment and Thermal Oxidation Protocol}

The remaining SWCNT dispersion was divided into 5 equal aliquots, each containing 120 $\mathrm{mL}$. Surfactant removal from the SWCNTs was examined by adding an equal volume of reagent to the specified aliquot. The reagents examined in the current study include acetone (ACTSWCNT), ethanol (EtOH-SWCNT), N,N-dimethlyacetamide (DMA-SWCNT), 1-cyclohexyl-2pyrrolidone (CHP-SWCNT), and acetonitrile (ACN-SWCNT). All solvents were purchased from Sigma-Aldrich with purity $\geq 99 \%$. The reagent treated dispersions were bath sonicated for $1 \mathrm{~h}$, then filtered onto a PTFE membrane to isolate the SWCNTs, which were rinsed with $50 \mathrm{~mL}$ of fresh solvent. The resulting bulk SWCNT samples were dried in a vacuum oven at $100^{\circ} \mathrm{C}$ for $1 \mathrm{~h}$. The purity of the reagent treated samples was characterized by SEM, Raman spectroscopy, TGA, XPS, and 4-point probe electrical measurements. Following characterization, each SWCNT sample (including both control samples and reagent treated samples) was thermally oxidized in air with a ramp-stop at $10^{\circ} \mathrm{C} / \mathrm{min}$ to $520^{\circ} \mathrm{C}$. Repeat characterization was performed and the results compared to the as-produced and P-SWCNT samples.

\section{Results and Discussion:}

The SWCNTs used in this work were synthesized in-house via laser vaporization, and their physical properties, including average length $(\sim 5 \mu \mathrm{m})$ [25] and diameter $(\sim 1.1 \mathrm{~nm})$ [26], have been well documented in the literature. The purity of the SWCNT starting material must be 
accurately characterized to provide a reference against which changes due to surfactant contamination can be compared, and to assess the efficacy of the reagent treatments. The SWCNT purity was characterized using optical absorbance spectroscopy (see Figure S1a), TGA (see Figure S1b), and XPS analysis (see Figure S2), and the defect content characterized via Raman spectroscopy (see Figure S1c). Based on these analyses, it is concluded that the PSWCNT reference material is comparable to a $100 \%$ pure SWCNT reference based on carbonaceous content from analysis of the optical absorbance spectrum [23], and contains $<1 \%$ metal catalyst content based on TGA. Surface analysis via XPS shows no measurable Ni or Co catalyst, confirming the TGA results. Additionally, analysis of the Raman spectra results in a $\mathrm{D} / \mathrm{G}$ ratio of 0.15 , which further demonstrates the relative quality of the SWCNT starting material $[27,28]$. Thus, the SWCNT starting material is sufficiently pure and nearly defect free, and can be used as a metric to compare the effects of residual surfactant and the efficiency of the proposed reagent-based surfactant removal method.

A number of reagents were qualitatively surveyed by mixing equal volumes of PSWCNTs dispersed in $2.0 \mathrm{wt} \%$ SDS with the desired reagent. Listed in Table 1 are the reagents surveyed, including various acids, bases, organic solvents, and salts. Although the effect of reagent mixing was almost immediately observed, the solution was bath sonicated for $1 \mathrm{~h}$ to ensure sufficient mixing and consistency between samples. Those reagents that interrupted the SDS:SWCNT interaction (i.e., caused SWCNT precipitation but did not change the SDS solubility) were of highest interest as these would allow the SWCNTs to be easily filtered from the bulk solution. Based on the qualitative analysis of approximately 20 reagents, five organic solvents yielded the preferred results, including acetone (ACT), ethanol (EtOH), N,Ndimethylacetamide (DMA), 1-cyclohexyl-2-pyrrolidone (CHP), and acetonitrile (ACN). Figure 
1a and b show representative photographs of the initial SWCNT dispersion in $2.0 \mathrm{wt} \%$ SDS and the desired reagent interaction, respectively. Reagents were eliminated from the study if they caused both the SWCNTs and SDS to precipitate, caused a visible side reaction indicated by a color change, or did not affect the SDS:SWCNT interaction (see Figure 1c-e, respectively). In general, the acids and bases were excluded from additional analysis because they exhibited an undesirable interaction with the SWCNTs, as shown in Table 1. Additionally, the influence of doping can convolute the interpretation of purification efficacy in the spectroscopic and electrical analysis.[29, 30] This qualitative reagent survey was conducted using a 1:1 (v:v) ratio to disrupt the SDS:SWCNT interaction for the selected reagents. The post-mixing sonication time and temperature were found to have little consequence on the efficacy of the reagent treatment. Thus, based on the results shown in Table 1, further characterization of the ACT-, EtOH-, DMA-, CHP-, and ACN-treated SWCNT samples was conducted to quantitatively determine how efficient each reagent was toward removing residual SDS from the SWCNTs. 
Table 1. The table provides a summary of the various reagents surveyed through qualitative visual inspection and their effect on the SDS:SWCNT interaction in solution.

\begin{tabular}{|c|c|c|c|c|c|}
\hline & Reagent & $\begin{array}{l}\text { SWCNT Only } \\
\text { Precipitation }\end{array}$ & $\begin{array}{c}S W C N T+S D S \\
\text { Precipitation } \\
\end{array}$ & $\begin{array}{c}\text { Visible } \\
\text { Reaction } \\
\end{array}$ & $\begin{array}{c}\text { No } \\
\text { Change }\end{array}$ \\
\hline \multirow{5}{*}{ Acids } & Hydrochloric acid & & & $\checkmark$ & \\
\hline & Nitric acid & & $\checkmark$ & & \\
\hline & Acetic acid & $\checkmark$ & & & \\
\hline & Sulfuric acid & & & $\checkmark$ & \\
\hline & Phosphoric acid & & $\checkmark$ & & \\
\hline \multirow{3}{*}{ Bases } & Sodium hydroxide & $\checkmark$ & & & \\
\hline & Potassium hydroxide & $\checkmark$ & & & \\
\hline & Hydrogen peroxide & & & & $\checkmark$ \\
\hline \multirow{8}{*}{ Organics } & Acetone & $\checkmark$ & & & \\
\hline & Acetonitrile & $\checkmark$ & & & \\
\hline & Ethanol & $\checkmark$ & & & \\
\hline & N,N-Dimethylacetamide & $\checkmark$ & & & \\
\hline & 1-Cyclohexyl-2-pyrrolidone & $\checkmark$ & & & \\
\hline & Dimethyl sulfoxide & $\checkmark$ & & & \\
\hline & Dodecanol & & $\checkmark$ & & \\
\hline & Chloroform & & $\checkmark$ & & \\
\hline Ionics & Sodium chloride & & $\checkmark$ & & \\
\hline
\end{tabular}

(a)

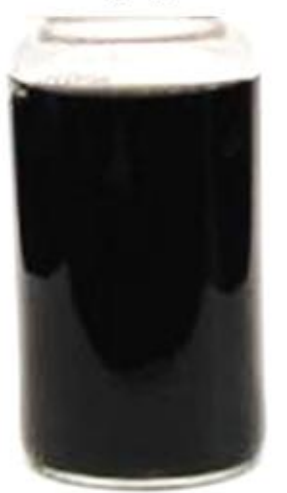

Starting

Dispersion (b)

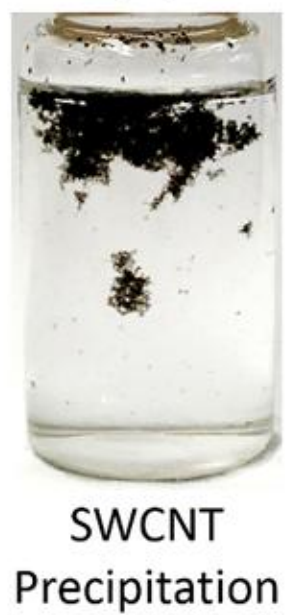

(c)

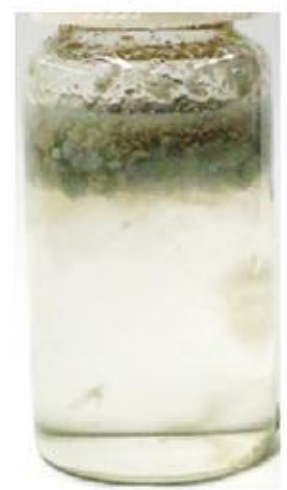

SWCNT + SDS

Precipitation

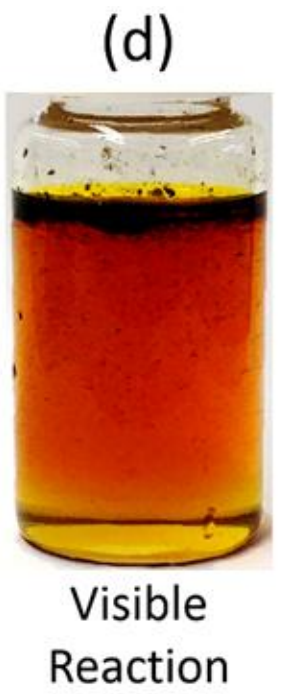

(d)

(e)

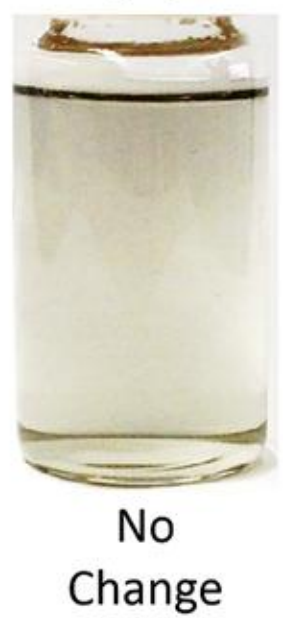

Figure 1. A photograph of (a) P-SWCNTs dispersed in aqueous $2.0 \mathrm{wt} \%$ SDS. Based on a qualitative screening of various acids, bases, organic solvents, and salts, a successful reagent will (b) interrupt the SWCNT:SDS interaction but not affect the solubility of the surfactant (e.g., P-SWCNTs + acetone), whereas an unsuccessful reagent will (c) cause both the SWCNTs and SDS to precipitate (e.g., P-SWCNTs + potassium hydroxide), (d) cause a side reaction indicated by color change (e.g., P-SWCNTs + sulfuric acid), or (e) have no effect on the SWCNT:SDS interaction (e.g., P-SWCNTs + hydrogen peroxide). 
SEM analysis was used as a rapid, but qualitative, measure of SWCNT purity. Approximately 25 micrographs of each sample were obtained from different regions and at different magnifications to ensure that the representative images shown herein are characteristic of the sample as a whole. Figure 2a shows a representative micrograph of the P-SWCNT control sample, which clearly indicates that the SWCNT surface morphology is free from carbonaceous and metal catalyst impurities. SEM analysis of the SDS-SWCNTs (see Figure 2b) reveals that there is a residual surfactant coating on the SWCNT surface, making the bundles more difficult to resolve. The surface morphology of the $\mathrm{H}_{2} \mathrm{O}-\mathrm{SWCNT}$ control sample (see Figure S3a) is nominally identical to that of the P-SWCNT control sample, confirming that the coating on the SDS-SWCNT sample is related to the residual surfactant and not carbonaceous impurities introduced during ultrasonication. The SEM analysis demonstrates that there is residual surfactant adsorbed to the SWCNT surface even though water washing removes bulk SDS from the sample. Therefore, development of the reagent-based purification method is necessary to remove the residual surfactant surface coatings and facilitate the complete purification of SWCNTs after exposure to SDS. 

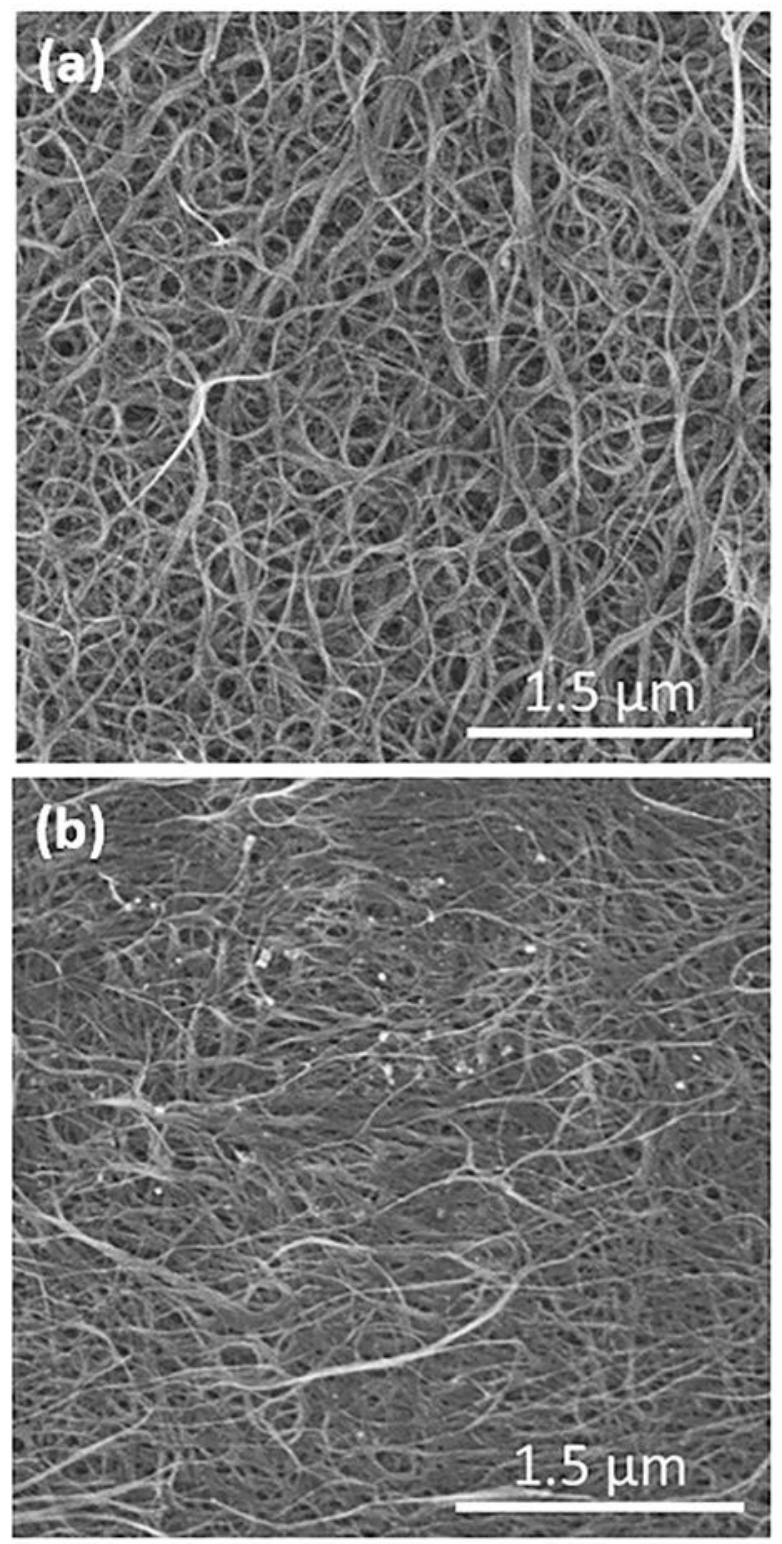

Figure 2. SEM analysis for the (a) P-SWCNT and (b) SDS-SWCNT (filtered from SDS and washed with $50 \mathrm{~mL}$ DI $\mathrm{H}_{2} \mathrm{O}$ ) control samples.

The purification process was scaled up so that SWCNT bulk papers could be fabricated after reagent treatment via vacuum filtration, with each sample having an areal density of $\sim 0.6$ $\mathrm{mg} / \mathrm{cm}^{2}$. The resulting SWCNT papers were rinsed with the appropriate solvent and dried in a vacuum oven at $100^{\circ} \mathrm{C}$ for $1 \mathrm{~h}$ to remove residual solvent/moisture. The filtration time and total processing time (filtration, rinse, etc.) for each reagent treated sample and the control samples 
are given in Table S1. Although concentrated $\mathrm{HCl}(37 \%)$ was excluded during the initial qualitative screening, the HCl-SWCNT control sample was fabricated using dilute acid (6 M) based on a previously established literature protocol.[20] A reduction in processing time was observed for the HCl-SWCNT samples ( 34\%); however, the improvement is not as significant compared to the reagent treatments due to the increased number of processing steps. The EtOH-, DMA-, and CHP-treatments yielded total processing times between $0.5-1 \mathrm{~h}$, which is $\geq 82 \%$ improvement compared to the SDS-SWCNT control sample. In particular, the acetone and acetonitrile treatments yielded the greatest reduction in processing time, which was decreased from nearly $6 \mathrm{~h}$ (for the SDS-SWCNT control) to less than $0.1 \mathrm{~h}$. Thus, compared to the SDSSWCNTs, the proposed reagent treatments reduce both the filtration and total processing time by up to $100 x$. It is important to note that filtration and processing times will vary based on the sample volume, concentration, membrane pore size, etc. However, these measured lab-scale times are intended to demonstrate that in addition to aiding in purification, the reagent treatments also significantly reduce processing times, thus enabling more efficient, large-scale SWCNT isolation from SDS dispersions.

The purity of the reagent treated samples was quantified relative to the P-SWCNT and SDS-SWCNT control samples using a combination of microscopy and spectroscopy. Representative SEM micrographs are shown in Figure 3 for the five reagent treated samples and the HCl-SWCNT reference sample. In general, the SWCNT morphology is apparent with no obvious surfactant coating for all reagent treated samples, and appears to be similar in all cases to the P-SWCNT control SEM shown in Figure 2a. With the exception of the ACT-SWCNT sample, the bundle size appears to increase (at least on the surface) compared to the P-SWCNT sample. After reagent treatment and subsequent thermal oxidation, the SWCNT morphology and 
purity appear to be nominally equivalent (see Figure S4). Analysis of the SEM data before and after reagent treatment show that the qualitative features are consistent with the P-SWCNT control, and no additional SWCNT ends are observed, suggesting that the SWCNT length distribution is unchanged. This analysis also demonstrates that the reagent treatment is effective compared to traditional water washing (refer to Figure 2b); however, additional characterization is required to determine the purity of the SWCNTs and whether residual SDS remains.
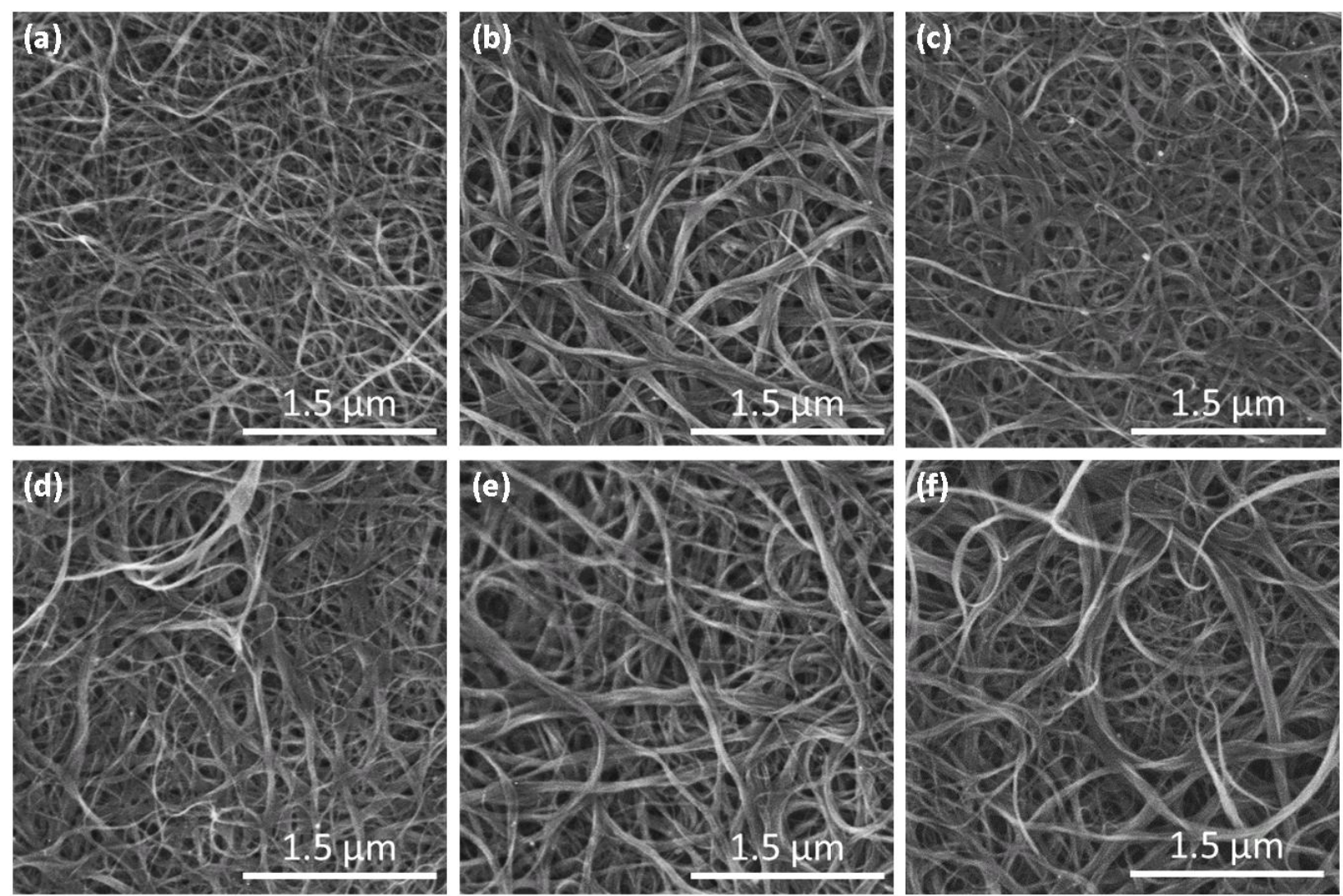

Figure 3. Representative SEM micrographs of the as-produced (a) ACT-, (b) EtOH-, (c) DMA-, (d) CHP-, (e) ACN-, and (f) HCl-treated SWCNT samples.

Thermogravimetric analysis (TGA) was performed to assess the decomposition characteristics of the reagent treated SWCNT samples compared to the P-SWCNT control. Approximately $0.5 \mathrm{mg}$ of each SWCNT sample was used to ensure consistency between 
measurements. Figure $\mathrm{S} 3 \mathrm{~b}$ shows the thermogram for the P-SWCNTs compared to the $\mathrm{H}_{2} \mathrm{O}$ SWCNT control sample, and confirms that processing has no effect on the decomposition temperature of the SWCNTs. Based on the first derivative weight loss curve (see Figure S3c), the P-SWCNT decomposition temperature is $640^{\circ} \mathrm{C}$. However, processing does seem to introduce some impurities, as evident by the low temperature decomposition features between $250-450^{\circ} \mathrm{C}$, and causes an increase in the residual mass. This is likely due to amorphization of the SWCNTs during ultrasonication and particulate matter released from the sonic horn tip during processing, respectively. Typically, ultracentrifugation would be used to remove these impurities prior to further work with the dispersion $[6-8,31]$; however, in the current study, the ultracentrifugation step was eliminated so that the SWCNT concentration could be precisely controlled. In addition, the decomposition of neat SDS and the SDS-SWCNT sample were compared to the P-SWCNT control sample. Figure S5 shows the rapid decomposition of the neat SDS at $\sim 200^{\circ} \mathrm{C}$ and a residual mass of $23 \%$. Likewise, the SDS-SWCNT sample shows a decrease in the SWCNT decomposition temperature to $513^{\circ} \mathrm{C}$ compared to the P-SWCNT control. The decrease in decomposition temperature in the SDS-SWCNT sample indicates the presence of residual surfactant, as does the small decomposition peak $\sim 200^{\circ} \mathrm{C}$, which matches that of neat SDS. As the SWCNT purity improves through removal of residual surfactant, it is expected that the maximum decomposition temperature will increase toward that of the $\mathrm{P}$ SWCNT control.

The reagent treated samples were characterized using TGA, and the weight loss results are shown in Figure 4a. In all cases (except CHP-SWCNT), the maximum decomposition temperature is increased above that of the SDS-SWCNT sample toward that of the P-SWCNT control. It is difficult to distinguish whether the low temperature decomposition is from residual 
SDS or amorphous carbon impurities from processing, and is most likely a combination of both. The as-produced reagent treated samples were subsequently subjected to a thermal oxidation treatment in air to $520^{\circ} \mathrm{C}$ using a ramp/stop of $10^{\circ} \mathrm{C} / \mathrm{min}$. Figure $4 \mathrm{~b}$ shows that the decomposition temperature of the SWCNT samples increases after thermal oxidation, and the impurities, which were observed at decomposition temperatures below $450^{\circ} \mathrm{C}$, are removed. An increase in residual mass is observed for both the SDS-SWCNT and CHP-SWCNT samples, which occurs due to the fact that the thermal oxidation temperature was greater than the SWCNT decomposition temperature of either as-produced sample. Although thermal oxidation produces an improvement (i.e., increase in SWCNT decomposition temperature) for all samples, the SDSSWCNT sample continues to exhibit the lowest decomposition temperature $\left(530^{\circ} \mathrm{C}\right)$ compared to the P-SWCNTs, and the reagent treated samples show increasing decomposition temperatures in order of ACT- $\left(544^{\circ} \mathrm{C}\right)<$ EtOH- $\left(552^{\circ} \mathrm{C}\right)<\mathrm{CHP}-\left(567^{\circ} \mathrm{C}\right)<$ DMA- $\left(582^{\circ} \mathrm{C}\right)<\mathrm{HCl}-\left(584^{\circ} \mathrm{C}\right)<$ ACN- $\left(592^{\circ} \mathrm{C}\right)$ SWCNTs. Thus, based on TGA analysis alone, thermal oxidation aids in SWCNT purification; however, the results are most improved when the samples are prepared with the reagent treatments. From this analysis, the acetonitrile treatment appears to be the most effective at removing residual SDS and produces a SWCNT sample most closely matched to the P-SWCNT control. 


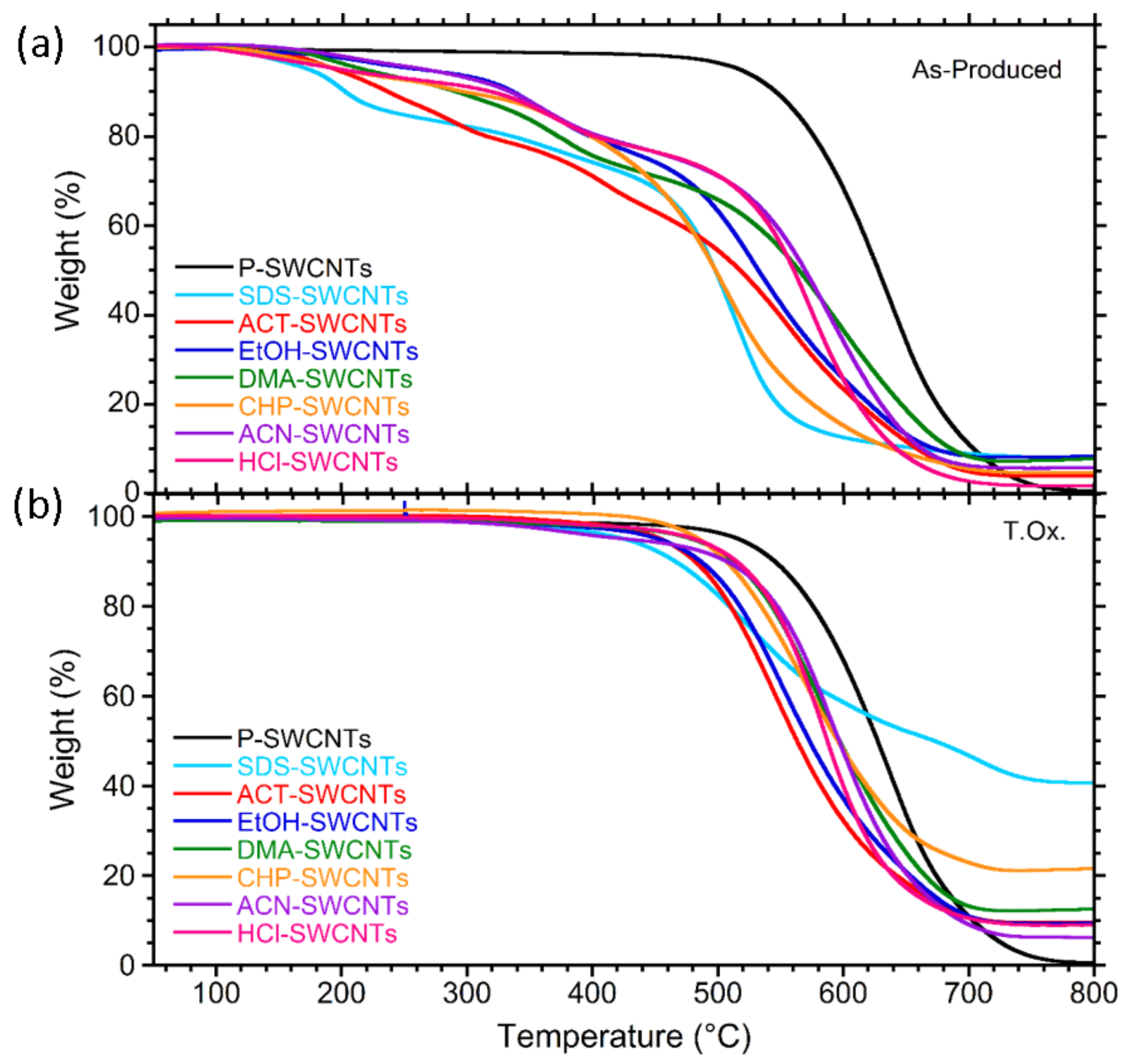

Figure 4. Thermogravimetric analysis (TGA) results of the weight loss as a function of temperature for (a) the asproduced reagent treated SWCNT samples and (b) after subsequent thermal oxidation (T. Ox.) in air with a $10^{\circ} \mathrm{C} / \mathrm{min} \mathrm{ramp} / \mathrm{stop}$ to $520^{\circ} \mathrm{C}$.

Raman spectroscopy was used to probe the quality of the reagent treated SWCNT samples. The full Raman spectra, which was acquired over a range from $100-3000 \mathrm{~cm}^{-1}$ to evaluate the characteristic SWCNT radial breathing mode (RBM), D, G, and $\mathrm{G}^{\prime}$ peaks, are shown in Figure S5 for the P-SWCNT and SDS-SWCNT controls, as well as the reagent treated 
samples. All data is normalized to the G peak maximum of the respective sample so that relative changes based on treatment condition may be easily discerned. SWCNT diameter can be characterized by analyzing the RBM, which is inversely related to peak position [32]. Comparison of the RBM for the P-SWCNT and $\mathrm{H}_{2} \mathrm{O}-\mathrm{SWCNT}$ controls (see Figure S3d), shows that there is a decrease in the peak intensity at $181 \mathrm{~cm}^{-1}$, suggesting some subtle degradation in the small diameter SWCNTs occurs due to the ultrasonication process. Analysis of the RBM in Figure S6 shows that neither the reagent treatment nor thermal oxidation has any additional effect on the SWCNT diameter distribution. Likewise, the Raman D peak intensity is not affected after processing. Thus, it is concluded that the SWCNT integrity is preserved, and the diameter and length distributions are unaffected by purification. Figure 5a shows a closer examination of the relative Raman G peak for the as-produced SWCNT samples. Compared to the P-SWCNT control, the SDS-SWCNT sample exhibits a significant suppression of the G peak due to the presence of the residual surfactant. In general, recovery in the $G$ peak lineshape is observed after reagent treatment, though some suppression is still observed in comparison to the P-SWCNT control. As shown in Figure 5b, the addition of thermal oxidation after reagent treatment causes near complete recovery in the BWF lineshape for all samples. The observed changes in the Raman G peak were quantified by analysis of the full width at half maximum (FWHM), which is determined by fitting the low frequency feature with the Breit-Wigner-Fano (BWF) lineshape and the high frequency feature with a single Lorentzian according to a previously reported method [33]. As shown in Figure 6, the P-SWCNT control has a FWHM of 86.5 (see dashed reference line). Exposure to SDS causes a reduction in the FWHM of the SDSSWCNT sample to 67.3. In general, the reagent treatment improves the FWHM to an average of 
$76.3 \pm 1.7$ (solid markers), and after subsequent thermal oxidation (open markers), all samples exhibit nominally equivalent FWHM with an average value of $82.4 \pm 0.7$.
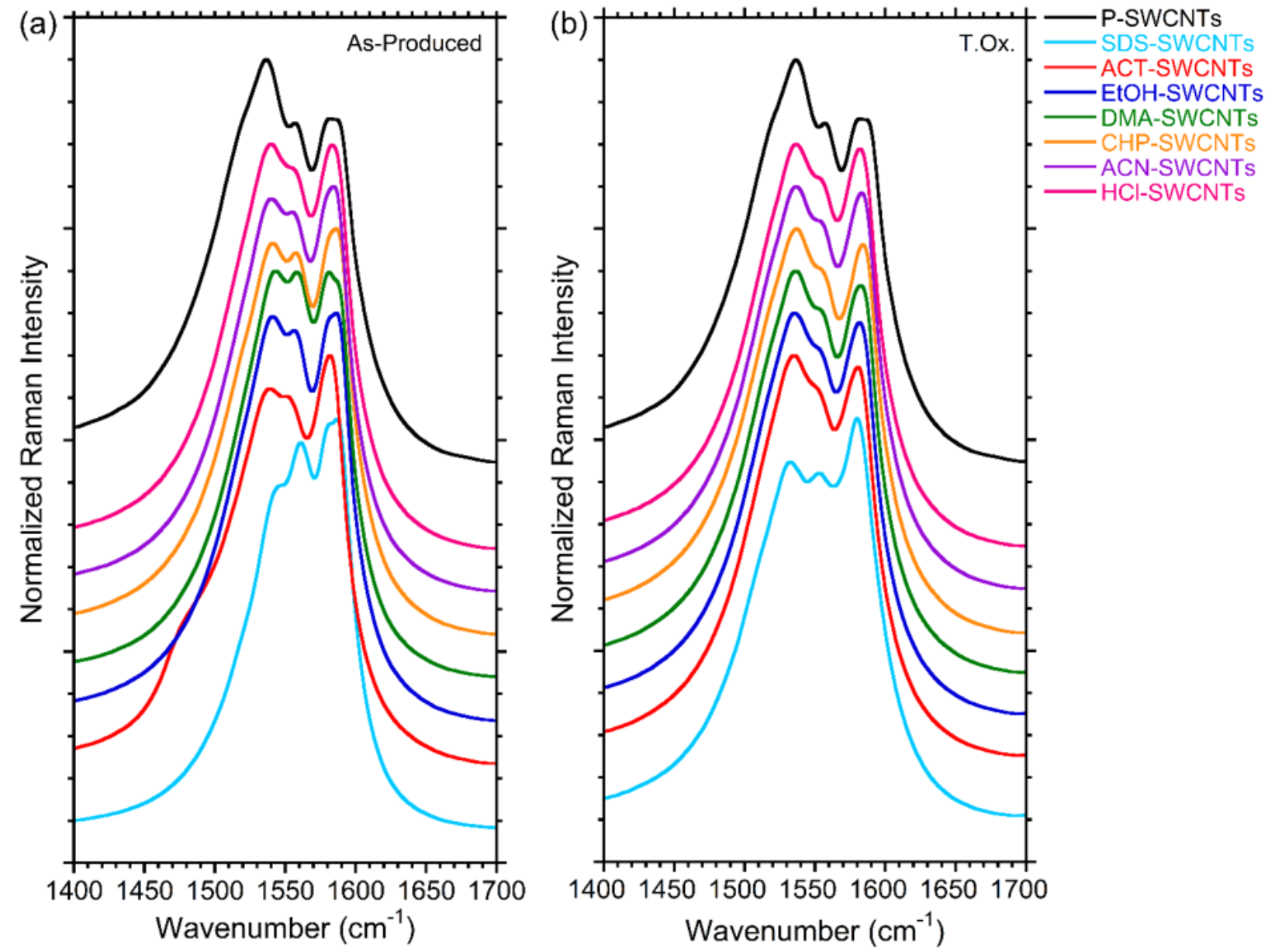

Figure 5. Analysis of the relative Raman G peak for the (a) as-produced and (b) thermally oxidized (T. Ox.) reagent treated samples compared to the P-SWCNT control (black curve). All data was normalized to the G peak maximum intensity for each sample.

Chemical doping of SWCNTs with surfactants has been reported previously, and can be distinguished based on changes observed in the G peak position and BWF feature [29, 34]. Suppression of the BWF lineshape in the presence of residual surfactant indicates that the SDS in the current study acts as a SWCNT dopant, which causes perturbations of the Fermi level due to charge exchange $[30,35,36]$. Recovery in the BWF lineshape after surfactant removal 
demonstrates that the doping is reversible and indicates that Raman spectroscopy can be used to assess purity in the reagent treated samples. Likewise, the Raman $\mathrm{G}^{\prime}$ peak position of the SWCNTs is sensitive to the presence of residual SDS, which manifests as an upshift in frequency compared to the P-SWCNT control $\left(2632 \mathrm{~cm}^{-1}\right.$ compared to $2625 \mathrm{~cm}^{-1}$, respectively, as shown in Figure S7a). The same doping effects described in the suppression of the BWF feature also contribute to the modulation in the $\mathrm{G}^{\prime}$ peak position, which has been previously demonstrated for both chemical [30] and electrochemical [37] doping of SWCNT structures. After reagent treatment, the $\mathrm{G}^{\prime}$ peak position is down-shifted toward that of the P-SWCNT control, with the greatest improvement observed for the ACT-SWCNT sample $\left(2623 \mathrm{~cm}^{-1}\right)$. Figure S7b shows that the $\mathrm{G}^{\prime}$ peak position of the remaining reagent treated samples is further recovered after the addition of a thermal oxidation treatment. Thus, it can be concluded from the Raman analysis, that the reagent treatment yields purity comparable to the P-SWCNT control, and the acetone and acetonitrile reagents continue to emerge as the most efficient treatments for disrupting the SDS:SWCNT interaction. 


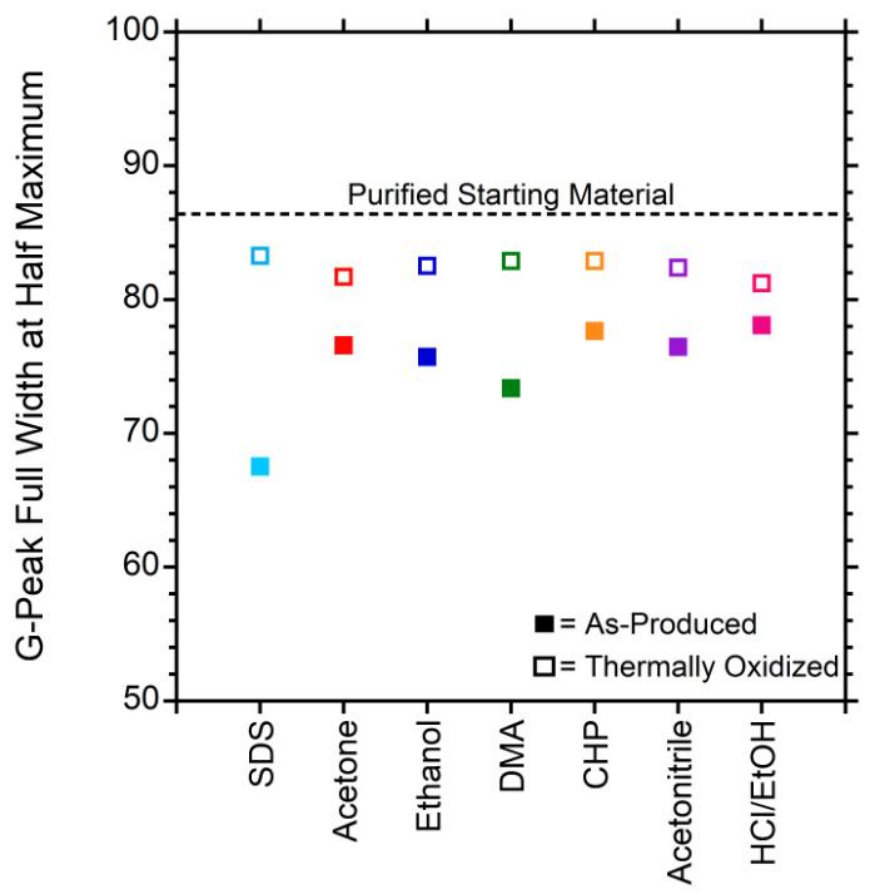

Figure 6. Analysis of the full width at half maximum (FWHM) for the Raman G peak after reagent treatment (solid markers) and subsequent thermal oxidation (open markers) compared to the P-SWCNT control (dashed line).

The electrical conductivity of the as-produced and thermally oxidized samples were evaluated and compared to the P-SWCNT control sample. As shown in Figure 7, the presence of residual SDS increases the conductivity of the SDS-SWCNT sample by $\sim 7.4 \mathrm{x}$ compared to the P-SWCNT control, again demonstrating that SDS acts as a chemical dopant with the SWCNTs. Although the enhanced conductivity afforded by the SDS-SWCNT sample can be useful in some applications, measuring the electrical conductivity of the SWCNTs can also be used as a metric to assess purification efficacy of the reagent treated samples. In general, there is a decrease in the electrical conductivity of the as-produced reagent treated samples compared to the SDSSWCNTs; however, the electrical conductivity is still greater than that of the P-SWCNT control. This is consistent with the Raman data, where the $\mathrm{G}^{\prime}$ peak position correlates with dopant concentration (i.e., lower $\mathrm{G}^{\prime}$ frequency suggests less doping and therefore lower electrical 
conductivity). The ACT-SWCNT sample shows the greatest recovery, which has an electrical conductivity of $4.23 \times 10^{4} \mathrm{~S} / \mathrm{m}$ compared to $2.86 \times 10^{4} \mathrm{~S} / \mathrm{m}$ for the P-SWCNT control. After thermal oxidation, the electrical conductivity of all reagent treated samples decreases to within $\pm 50 \%$ of the P-SWCNT control value. Although previous reports have shown increased electrical conductivity with removal of surfactant via nitric acid treatment, those increases may have resulted from nitric acid doping and no quantitative surfactant removal evidence was provided $[9,10]$. While the effect of residual surfactant on device performance has not been fully characterized, it has been reported that it has little to no effect on the field-effect mobility of single SWCNT field effect transistor devices [18]. However, this does not account for surfactant effects in thin-films or bulk papers, where the SWCNT network will contain a mixture of metallic and semiconducting electronic-types with varying residual SDS concentration. Thus, the ability to controllably remove SDS from SWCNTs to recover the intrinsic electrical properties will allow for reproducible device fabrication through post-processing purification in situations where the use of surfactant is necessary. 


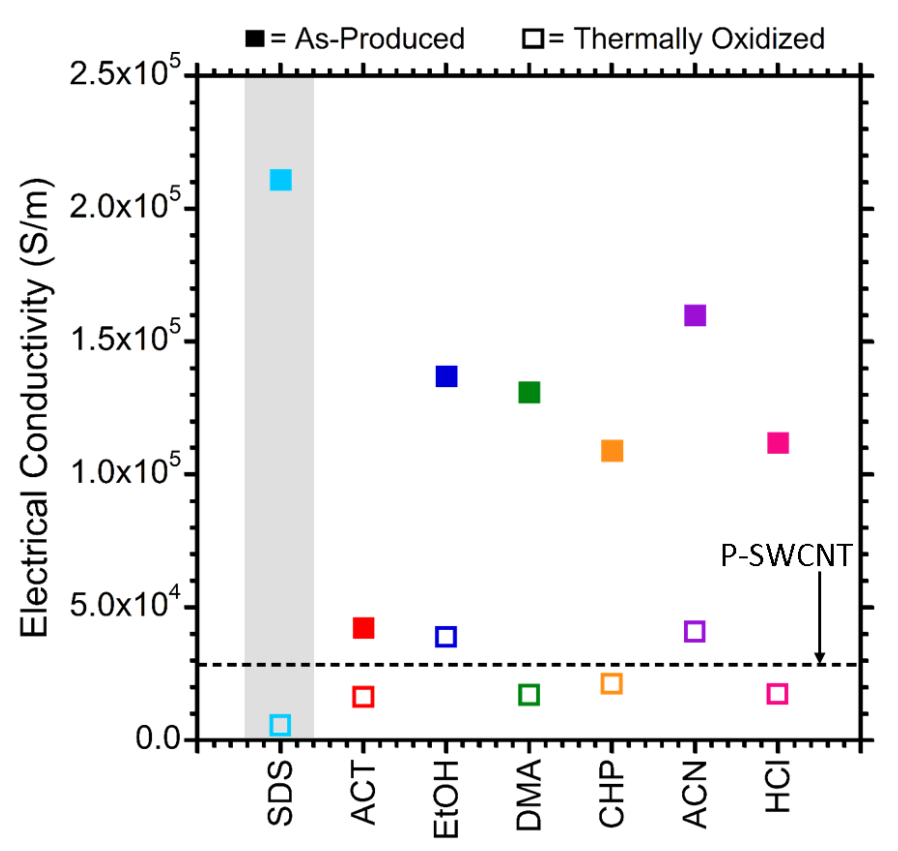

Figure 7. Electrical conductivity for the as-produced (closed markers) and thermally oxidized (T. Ox., open markers) SDS-SWCNT and the reagent treated SWCNT samples compared to the P-SWCNT control (dashed line).

Based on the results of the SEM, TGA, Raman, and electrical conductivity analysis, the acetone and acetonitrile treatments emerge as the most efficient for SDS removal from SWCNTs. Thus, these samples were analyzed with x-ray photoelectron spectroscopy (XPS) to determine how much surfactant remains after treatment. The XPS measurements were acquired with the flood gun on in order to minimize charging. A non-linear Shirley background subtraction was used to fit the individual spectra, which are shown in Figure S8. The area under the curve was used to determine the atomic concentrations of $\mathrm{C}, \mathrm{O}, \mathrm{Na}$, and $\mathrm{S}$ in each sample. As shown in Table 2, the P-SWCNT control showed no evidence of Na or S present, therefore, any contribution of these elements in the SDS-SWCNT control sample or ACT- and ACNSWCNT reagent treated samples is due to the presence of residual surfactant. A 3x increase in the atomic $\mathrm{O}$ concentration, and $\sim 5-6 \mathrm{x}$ increase in $\mathrm{Na}$ and $\mathrm{S}$ concentration is observed for both the as-produced and thermally oxidized SDS-SWCNT samples compared to the P-SWCNT 
control. This corroborates the TGA, Raman, and electrical analysis, which demonstrates that residual SDS cannot be removed through thermal treatment alone. The acetone and acetonitrile treatments improve the purity of the SWCNTs more than thermal oxidation; however, when the reagent treatment is used in combination with thermal oxidation, nearly all of the residual SDS is removed from the SWCNT samples. Following the acetone treatment, no residual S is detected by XPS analysis, and only 0.3 at.\% remains in the acetonitrile treated sample. Both the ACTand ACN- treated SWCNTs have $\sim 0.3$ at.\% Na remaining after thermal oxidation, which is more difficult to remove due to the nature of the Na-C-O bonding. Likewise, the atomic $\mathrm{O}$ concentration is reduced to (or below) that of the P-SWCNT control sample. Although the presence of residual surfactant in the SDS-SWCNT samples caused a $\sim 7 \mathrm{x}$ increase in the O/C ratio compared to the P-SWCNT control (0.2 compared to 0.03, respectively), the reagent treatment coupled with thermal oxidation restored this value to 0.03 . The analysis presented herein, demonstrates that acetone or acetonitrile reagent treatment in conjunction with thermal oxidation results in SWCNTs that have $>95 \%$ carbonaceous purity.

Table 2. The atomic percentage of $\mathrm{C}, \mathrm{O}, \mathrm{Na}$, and $\mathrm{S}$ was characterized using XPS analysis for the P-SWCNT and SDS-SWCNT control samples, and the ACT- and ACN- treated SWCNT samples. This analysis was conducted on both the as-produced (-AP) and thermally oxidized (-TOx) SWCNTs.

\begin{tabular}{ccccccc}
\hline SWCNT Sample & $C$ & $O$ & $N a$ & $S$ & Other & $O / C$ \\
\hline \hline P-AP & 96.8 & 3.2 & -- & -- & -- & 0.03 \\
SDS-AP & 77.7 & 13.4 & 6.7 & 2.0 & 0.2 & 0.17 \\
SDS-TOx & 73.5 & 14.6 & 5.5 & 5.8 & 0.6 & 0.20 \\
ACT-AP & 88.8 & 6.8 & 1.1 & 0.5 & 2.8 & 0.07 \\
ACT-TOx & 96.6 & 3.2 & 0.2 & -- & -- & 0.03 \\
ACN-AP & 95.5 & 3.1 & 0.2 & 0.8 & 0.4 & 0.03 \\
ACN-TOx & 97.5 & 1.9 & 0.3 & 0.3 & -- & 0.02 \\
\hline
\end{tabular}


The reagent treatment described herein provides a simple and efficient means to remove SDS from aqueous dispersions of SWCNTs, and enables a method for the intrinsic SWCNT properties to be investigated after surfactant processing. Although treatment with either acetone or acetonitrile is most effective at removing SDS from the SWCNTs, these results demonstrate that the reagent treatment coupled with thermal oxidation produces the greatest improvement in purity after exposure to the surfactant. The data also shows that thermal oxidation alone is not effective in SWCNT purification without the use of the reagent treatment. It should be noted that the two reagents (acetone and acetonitrile), which provide the greatest improvement in purity, also yielded the greatest improvement in processing time. Therefore, the method of purification via reagent treatment coupled with thermal oxidation yields potential for large volume SWCNT processing. In particular, this work has significant implication for those pursuing SWCNT chirality separations, as a majority of SWCNT separation techniques utilize surfactants and can benefit from this facile, scalable, and efficient method of post-processing SWCNT purification.

\section{Conclusions:}

A reagent-based treatment method has been demonstrated for the removal of SDS from bulk quantities of SWCNTs in aqueous dispersion. After qualitative survey of various acids, bases, organic solvents, and ionic solutions, it was determined that five organic solvents, including acetone, ethanol, DMA, CHP, and acetonitrile, disrupted the SDS:SWCNT interaction and allowed for the facile isolation of purified SWCNTs through vacuum filtration. Quantitative analysis via TGA, Raman spectroscopy, 4-pt. probe electrical measurement, and XPS has demonstrated that the acetone and acetonitrile treatments produce the greatest improvement in 
SWCNT purity, which can be further improved with the addition of a thermal oxidation treatment. It was also demonstrated that the acetone and acetonitrile reagent treatments resulted in a 100x improvement (i.e., reduction) in processing time, which yields SWCNTs that are free from residual surfactant and have a carbonaceous purity $>95 \%$. In the current work, $1 \mathrm{mg}$ of SWCNT material in $120 \mathrm{~mL}$ was processed at a time, however, it is expected that the reagent treatment can be scaled to gram quantities in several liters of volume (or larger). Thus, the ability to quickly isolate large quantities of highly pure SWCNTs from aqueous SDS dispersions through the use of the reagent treatment method is expected to impact applications such as spectroscopy, chirality separations, and device fabrication.

\section{Acknowledgements:}

The authors gratefully acknowledge funding from the U.S. Government, the Defense Threat Reduction Agency (DTRA) under Grant HDTRA-1-10-1-0122, and the Office of Naval Research under Grant N00014-15-1-2720. C. D. Cress and E. Cleveland acknowledge funding from the Office of Naval Research 6.1 Base Funding. This research was performed while S.W. Schmucker held a National Research Council Associateship Award at the Naval Research Laboratory. This material is based upon work funded in whole or in party by the U.S. Government, and any opinions, findings, conclusions, or recommendations expressed in this material are those of the author(s) and do not necessarily reflect the views of the U.S. Government.

\section{$\underline{\text { References: }}$}

[1] Bachilo, S. M.; Strano, M. S.; Kittrell, C.; Hauge, R. H.; Smalley, R. E.; Weisman, R. B. StructureAssigned Optical Spectra of Single-Walled Carbon Nanotubes. Science, 2002, 298, 2361-2366. 
[2] Haroz, E. H.; Duque, J. G.; Lu, B. Y.; Nikolaev, P.; Arepalli, S.; Hauge, R. H.; Doorn, S. K.; Kono, J. Unique Origin of Colors of Armchair Carbon Nanotubes. J Am Chem Soc, 2012, 134, 4461-4464.

[3] Jeong, S. H.; Kim, K. K.; Jeong, S. J.; An, K. H.; Lee, S. H.; Lee, Y. H. Optical Absorption Spectroscopy for Determining Carbon Nanotube Concentration in Solution. Synthetic Met, 2007, 157, 570-574.

[4] Moore, V. C.; Strano, M. S.; Haroz, E. H.; Hauge, R. H.; Smalley, R. E.; Schmidt, J.; Talmon, Y. Individually Suspended Single-Walled Carbon Nanotubes in Various Surfactants. Nano Lett, 2003, 3, 1379-1382.

[5] Yanagi, K.; Miyata, Y.; Tanaka, T.; Fujii, S.; Nishide, D.; Kataura, H. Colors of Carbon Nanotubes. Diam Relat Mater, 2009, 18, 935-939.

[6] Hersam, M. C. Progress Towards Monodisperse Single-Walled Carbon Nanotubes. Nat Nanotechnol, 2008, 3, 387-394.

[7] Tanaka, T.; Liu, H. P.; Fujii, S.; Kataura, H. From Metal/Semiconductor Separation to SingleChirality Separation of Single-Wall Carbon Nanotubes Using Gel. Phys Status Solidi-R, 2011, 5, 301-306.

[8] Tanaka, T.; Urabe, Y.; Nishide, D.; Liu, H. P.; Asano, S.; Nishiyama, S.; Kataura, H. Metal/Semiconductor Separation of Single-Wall Carbon Nanotubes by Selective Adsorption and Desorption for Agarose Gel. Phys Status Solidi B, 2010, 247, 2867-2870.

[9] Gao, J.; Wang, W. Y.; Cui, L. J.; Chen, L. T.; Hu, X. Y.; Li, H.; Geng, H. Z. Effect of Different Concentrations of Nitric Acid on the Conductivity of Single-Walled Carbon Nanotube Transparent Films. Advanced Materials Research, 2013, 658, 3-7.

[10] Geng, H. Z.; Kim, K. K.; So, K. P.; Lee, Y. S.; Chang, Y.; Lee, Y. H. Effect of Acid Treatment on Carbon Nanotube-Based Flexible Transparent Conducting Films. J Am Chem Soc, 2007, 129, 7758-+.

[11] Tyler, T. P.; Brock, R. E.; Karmel, H. J.; Marks, T. J.; Hersam, M. C. Electronically Monodisperse Single-Walled Carbon Nanotube Thin Films as Transparent Conducting Anodes in Organic Photovoltaic Devices. Adv Energy Mater, 2011, 1, 785-791.

[12] Jaber-Ansari, L.; Iddir, H.; Curtiss, L. A.; Hersam, M. C. Influence of Electronic Type Purity on the Lithiation of Single-Walled Carbon Nanotubes. Acs Nano, 2014, 8, 2399-2409.

[13] Wu, Z. C.; Chen, Z. H.; Du, X.; Logan, J. M.; Sippel, J.; Nikolou, M.; Kamaras, K.; Reynolds, J. R.; Tanner, D. B.; Hebard, A. F.; Rinzler, A. G. Transparent, Conductive Carbon Nanotube Films. Science, 2004, 305, 1273-1276.

[14] Zhou, Y. X.; Hu, L. B.; Gruner, G. A Method of Printing Carbon Nanotube Thin Films. Appl Phys Lett, 2006, 88.

[15] Park, J. G.; Smithyman, J.; Lin, C. Y.; Cooke, A.; Kismarahardja, A. W.; Li, S.; Liang, R.; Brooks, J. S.; Zhang, C.; Wang, B. Effects of Surfactants and Alignment on the Physical Properties of Single-Walled Carbon Nanotube Buckypaper. J Appl Phys, 2009, 106.

[16] Shastry, T. A.; Seo, J. W. T.; Lopez, J. J.; Arnold, H. N.; Kelter, J. Z.; Sangwan, V. K.; Lauhon, L. J.; Marks, T. J.; Hersam, M. C. Large-Area, Electronically Monodisperse, Aligned Single-Walled Carbon Nanotube Thin Films Fabricated by Evaporation-Driven Self-Assembly. Small, 2013, 9, 45-51.

[17] Wang, J. P.; Sun, J.; Gao, L.; Liu, Y. Q.; Wang, Y.; Zhang, J.; Kajiura, H.; Li, Y. M.; Noda, K. Improving the Conductivity of Single-Walled Carbon Nanotubes Films by Heat Treatment. J Alloy Compd, 2009, 485, 456-461.

[18] Kane, A. A.; Ford, A. C.; Nissen, A.; Krafcik, K. L.; Leonard, F. Etching of Surfactant from SolutionProcessed, Type-Separated Carbon Nanotubes and Impact on Device Behavior. Acs Nano, 2014, 8, 24772485.

[19] Ng, M. H. A.; Hartadi, L. T.; Tan, H.; Poa, C. H. P. Efficient Coating of Transparent and Conductive Carbon Nanotube Thin Films on Plastic Substrates. Nanotechnology, 2008, 19. 
[20] Lukaszczuk, P.; Rummeli, M. H.; Knupfer, M.; Kalenczuk, R. J.; Borowiak-Palen, E. Surfactant Free Fractions of Metallic and Semiconducting Single-Walled Carbon Nanotubes Via Optimised Gel Chromatography. Mater Res Bull, 2012, 47, 687-691.

[21] Wang, J. P.; Sun, J.; Gao, L.; Wang, Y.; Zhang, J.; Kajiura, H.; Li, Y. M.; Noda, K. Removal of the Residual Surfactants in Transparent and Conductive Single-Walled Carbon Nanotube Films. J Phys Chem C, 2009, 113, 17685-17690.

[22] Landi, B. J.; Ruf, H. J.; Worman, J. J.; Raffaelle, R. P. Effects of Alkyl Amide Solvents on the Dispersion of Single-Wall Carbon Nanotubes. J Phys Chem B, 2004, 108, 17089-17095.

[23] Landi, B. J.; Ruf, H. J.; Evans, C. M.; Cress, C. D.; Raffaelle, R. P. Purity Assessment of Single-Wall Carbon Nanotubes, Using Optical Absorption Spectroscopy. J Phys Chem B, 2005, 109, 9952-9965.

[24] Landi, B. J.; Cress, C. D.; Evans, C. M.; Raffaelle, R. P. Thermal Oxidation Profiling of Single-Walled Carbon Nanotubes. Chem Mater, 2005, 17, 6819-6834.

[25] Landi, B. J.; Ganter, M. J.; Cress, C. D.; DiLeo, R. A.; Raffaelle, R. P. Carbon Nanotubes for Lithium Ion Batteries. Energ Environ Sci, 2009, 2, 638-654.

[26] Rossi, J. E.; Cress, C. D.; Merrill, A.; Soule, K. J.; Cox, N. D.; Landi, B. J. Intrinsic Diameter Dependent Degradation of Single-Wall Carbon Nanotubes from Ion Irradiation. Carbon, 2015, 81, 488496.

[27] Dresselhaus, M. S.; Jorio, A.; Saito, R. Characterizing Graphene, Graphite, and Carbon Nanotubes by Raman Spectroscopy. Annu Rev Conden Ma P, 2010, 1, 89-108.

[28] Dresselhaus, M. S.; Jorio, A.; Souza, A. G.; Saito, R. Defect Characterization in Graphene and Carbon Nanotubes Using Raman Spectroscopy. Philos T R Soc A, 2010, 368, 5355-5377.

[29] Blackburn, J. L.; Engtrakul, C.; McDonald, T. J.; Dillon, A. C.; Heben, M. J. Effects of Surfactant and Boron Doping on the Bwf Feature in the Raman Spectrum of Single-Wall Carbon Nanotube Aqueous Dispersions. J Phys Chem B, 2006, 110, 25551-25558.

[30] Puchades, I.; Lawlor, C. C.; Schauerman, C. M.; Bucossi, A. R.; Rossi, J. E.; Cox, N. D.; Landi, B. J. Mechanism of Chemical Doping in Electronic-Type-Separated Single Wall Carbon Nanotubes Towards High Electrical Conductivity. J Mater Chem C, 2015, 3, 10256-10266.

[31] Blackburn, J. L.; Barnes, T. M.; Beard, M. C.; Kim, Y. H.; Tenent, R. C.; McDonald, T. J.; To, B.; Coutts, T. J.; Heben, M. J. Transparent Conductive Single-Walled Carbon Nanotube Networks with Precisely Tunable Ratios of Semiconducting and Metallic Nanotubes. Acs Nano, 2008, 2, 1266-1274.

[32] Bandow, S.; Asaka, S.; Saito, Y.; Rao, A. M.; Grigorian, L.; Richter, E.; Eklund, P. C. Effect of the Growth Temperature on the Diameter Distribution and Chirality of Single-Wall Carbon Nanotubes. Phys Rev Lett, 1998, 80, 3779-3782.

[33] Brown, S. D. M.; Jorio, A.; Corio, P.; Dresselhaus, M. S.; Dresselhaus, G.; Saito, R.; Kneipp, K. Origin of the Breit-Wigner-Fano Lineshape of the Tangential G-Band Feature of Metallic Carbon Nanotubes. Phys Rev B, 2001, 63.

[34] Samarajeewa, D. R.; Dieckmann, G. R.; Nielsen, S. O.; Musselman, I. H. Doping Single-Walled Carbon Nanotubes with Surfactant Peptides Containing Electron-Donor Substituents and Nitrogen Heterocycles. Carbon, 2013, 57, 88-98.

[35] Jorio, A.; Dresselhaus, M. S.; Saito, R.; Dresselhaus, G. F., Raman Spectroscopy in Graphene Related Systems; Wiley-VCH: Weinheim, Germany, 2011.

[36] Rao, A. M.; Eklund, P. C.; Bandow, S.; Thess, A.; Smalley, R. E. Evidence for Charge Transfer in Doped Carbon Nanotube Bundles from Raman Scattering. Nature, 1997, 388, 257-259.

[37] Kalbac, M.; Kavan, L.; Farhat, H.; Kong, J.; Dresselhaus, M. S. Large Variety of Behaviors for the Raman G ' Mode of Single Walled Carbon Nanotubes Upon Electrochemical Gating Arising from Different (N,M) of Individual Nanotubes. J Phys Chem C, 2009, 113, 1751-1757. 


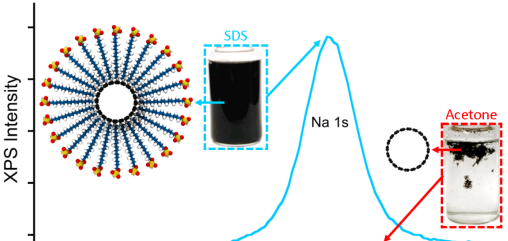

